\title{
ON THE CRYPTOBIOSIS OF LYMNAEA CUBENSIS, THE SNAIL VECTOR OF FASCIOLA HEPATICA IN PUERTO RICO ${ }^{1,2}$
}

Cryptobiosis, a term coined by David Keilin, ${ }^{3}$ describes a death-like state of almost complete dehydration of certain primitive organisms followed by their renewed biological activity as a result of moistening and rehydration. ${ }^{4}$ Cryptobiosis is observed in some species of fresh-water snails.

Kendall ${ }^{5}$ was the first researcher to report the occurrence of such phenomenon in Lymnaea truncatula, the snail intermediate host of $F$. hepatica, designating it "aestivation". $\mathrm{He}^{6}$ described the effect of lymnaeid-snail cryptobiosis upon the epidemiology of fascioliasis in the temperate zone, but not under tropical conditions where high soil temperatures and almost complete dehydration of snail habitats occur. Observations on lymnaeidsnail cryptobiosis under conditions in Puerto Rico follow.

Due to heavy rainfall and floods, favorable conditions for increased population densities of lymnaeid snails occurred during October, November and December of 1970 on nine farms studied in the Dorado-Toa Baja area. Test areas negative for snails in 1970 were found densely populated by $L$. cubensis and heavily infested with $F$. hepatica in February and March 1971. Habitats on the nine farms were then marked with bamboo stakes and examined monthly for the presence of snails.

By late April, 1971, test areas started to become progressively drier, and the snail population simultaneously started to decrease. The ground became cracked and "bone-dry" five months later and no snails could be found in the majority of the sites. Ten dried mud slabs about $12 \times 12 \times 11 / 2$ inches were collected from test sites at each of the nine farms. Each slab was placed in a separate plastic container and brought to the laboratory. Just enough dechlorinated tap water was added to each container to cover the sample. All containers, fitted with fine wire-mesh lids, were kept for two weeks under propitious conditions for optimum snail growth and development, then checked for presence of snails. Samples were negative for snails from two farms. From one to three of the 10 mud samples from each

${ }^{1}$ Manuscript submitted to Editorial Board November 6, 1974.

2 Thanks are expressed to Mr. M. Santana, Department of Agriculture of Puerto Rico, for his technical assistance.

${ }^{8}$ Keilin, D., The problem of anabiosis or latent life: History and current concept. Proc. Roy. Soc. of London, Series B-Biological Sciences 150 (939) : 149-191, 1959.

- Crowe, J. H., and Cooper, Jr., A. F., Cryptobiosis, Scientific American, 225 (6): 30-36, 1971.

- Kendall, S. B., Bionomics of Lymnaea truncatula and the parthenitae of Fasciola hepatica under drought conditions, J. Helminthology 23 (1-2): 57-68, 1949.

- L loc. cit. 
of the other 7 farms were positive. Each positive sample contained from one to four snails, 2 to $4 \mathrm{~mm}$ in length. The average snail density was 24 in March, decreased abruptly to 0.03 in May, to none in June on farm No. 2. Three of 10 mud samples taken September, 1971 revealed live snails in October, 1971.

It could not be ascertained from this series of investigations whether the snails recovered from the wetted mud samples were newly hatched individuals from egg-masses contained in the dry slabs or cryptobiotical survivors of the drought situation.

To further our information in this subject matter area, 10 to 12 adult snails were introduced into each of $\mathbf{1 2}$ tall glasses containing mud and algae, where they deposited egg-masses. All snails were removed on the 4th day and the water carefully decanted from the glasses. Mud in the glasses dried within a month. Dechlorinated tap-water was subsequently added to the glasses which then were checked daily for presence of snails. All were negative. Furthermore, no indication of embryonic development was noted in egg-masses lifted from the culture glasses. Additionally, 10 to 12 snails of various ages were introduced into 12 other glasses. Supplemental feed was added and the snails were allowed to reproduce. The cultures in the glasses were allowed to dry out gradually. Each dried-out, compacted mud-culture was removed from its glass two months later and sliced transversely into three inch thick slabs. These were placed separately in Petri dishes and dechlorinated tap-water added. Very small snails, about 1 to 2 $\mathrm{mm}$ long, were observed in half the 12 top-portion slices. None were observed in the 24 slices from the lower levels.

These preliminary findings tend to underscore the expected sensitivity of $L$. cubensis ova to dehydration. The observations apparently also tend to indicate that only very small snails can undergo cryptobiosis, and that these are within one inch of the surface of the ground.

Under laboratory conditions, such young snails probably are not infected with $F$. hepatica after their cryptobiotic stage. However, under field conditions, small snails may serve as the nucleus for the snail population in the next rainy season. The possibility of carryover of infestation under such field condition indicates need for definitive studies of pertinent ecologic factors.

J. Chiriboga and L. S. Ritchie, Puerto Rico Nuclear Center D. de León, Agricultural Experiment Station 